\title{
Predictors of drinking immediacy following daily sadness: An application of survival analysis to experience sampling data
}

\author{
Andrea M. Hussong* \\ Department of Psychology, CB\#3270 Davie Hall, University of North Carolina, Chapel Hill, NC \\ 27599-3270, United States
}

\begin{abstract}
Previous studies of daily assessments show modest mood-drinking covariation as a function of gender and coping motives; however previous analyses also assume a fixed interval across all individuals in the onset of drinking following negative mood. The current study used survival analysis and experience sampling methods to test whether gender and coping motives predicted shorter sadness-to-drinking intervals among those with greater alcohol-related drinking consequences. A sample of 85 college students (46\% male; $78 \%$ Caucasian) completed daily assessments over 28 days. Survival analyses showed that women drank more on days following elevated sadness when they reported being motivated to drink to cope and having experienced alcohol-related consequences. For men, the two groups showing greater drinking risk following days of elevated sadness did not report alcohol-related consequences, with those reporting the presence of coping motives showing the greatest risk. Implications of these findings for selfmedication mechanisms are discussed.
\end{abstract}

\section{Keywords}

College drinking; Self-medication; Experience sampling

Experience sampling methods are increasingly used to evaluate variants of the selfmedication hypothesis of heavy drinking (e.g., Hussong, Hicks, Levy, \& Curran, 2001; Swendsen, 2000). The self-medication hypothesis posits that heavy drinking is motivated by increased negative affect in an effort to alleviate distress (Greeley \& Oei, 1999). ${ }^{1}$ Experience sampling methods permit time-linked data collection such that time-specific measures of affect and drinking can be sampled from the constant stream of affective experiences and the more discrete events of drinking, respectively, throughout a day, week or month (see Tennen \& Affleck, 2002, for a review of the technique). Temporal precedence can then be established to determine whether elevations in sadness typically occur prior to heavy bouts of drinking as predicted by the self-medication hypothesis. The ability to establish such temporal precedence on a microanalytic scale permits us to isolate the similarly microanalytic predictions of the self-medication hypothesis from alternative explanations for associations between affect and alcohol use. In contrast, typical longitudinal designs that assess affect and drinking over longer intervals (i.e., over the past year or month as measured on two occasions) cannot differentiate associations between the two that are due to the mechanism of self-medication versus the mechanism of shared precursors (i.e.,

\footnotetext{
(c) 2006 Elsevier Ltd. All rights reserved.

*Tel.: +1 919962 6593; fax: +1 919962 2537. hussong@unc.edu.

${ }^{1}$ Although Baker, Piper, McCarthy, Majeskie, and Fiore (2004) indicate that high levels of negative mood and sadness may not be necessary for self-medication processes to operate, this model is perhaps most applicable to individuals with long-term addiction rather than the college-sample of interest here.
} 
affect and alcohol use having a single shared etiology, such as genetic mediation or family disturbance, that leads to both) because the element of time that in part distinguishes the self-medication hypothesis is not represented in such methods (see Hussong et al., 2001). ${ }^{2}$

Using experience sampling and daily diary methods, recent studies show mixed support for a modest relation between negative affect and drinking behavior. In a sample of moderate drinkers reporting daily mood and alcohol use, Armeli, Tennen, Affleck, and Kranzler (2000) found that individuals were more likely to drink on days characterized by greater sadness. Similarly, Flynn (2000) found greater drinking on days when greater depression was reported in a sample of college students and Swendsen et al. (2000) found greater drinking on days when greater nervousness was reported in a community sample of adults. However, other studies report no main effect of daily sadness on drinking (Hussong, Galloway, \& Feagans, 2004) or even a negative association between daily mood and drinking (where mood was assessed as anxiety, Steptoe \& Wardle, 1999).

These studies of daily mood-drinking relations largely assess intra-day associations (although some test these associations within a 1-day lag). However, the optimal interval over which elevated sadness may predict subsequent drinking is not known and indeed is likely to vary over individuals. In such cases, weekday distress may build up until the weekend when drinking may be particularly elevated given a stressful week. For others, drinking may be less responsive to social constraints and more immediate in response to negative affect such that work, social and other responsibilities are ignored and drinking opportunities are directly sought as a means of coping when acute experiences of distress occur. Clearly, such posited individual variation in drinking responses to acute distress suggests that more immediate responses are more problematic and perhaps more indicative of a lack of control over drinking, a style of drinking that is likely to garner greater negative consequences and reflect an immature style of emotional regulation. Thus, traditional statistical techniques, like hierarchical or mixed model, that require a fixed interval over individuals for testing mood-drinking relations may be limited in testing this hypothesized effect.

Other markers of vulnerability for self-medication identified in previous literature may also predict greater risk for drinking in the days subsequent to high levels of sadness. For example, Cooper, Russell, Skinner, Frone, and Mudar (1992) posit and find support for a stronger relation between negative emotion and drinking among those who are more motivated to drink so as to cope with distress as well among men as opposed to women. The role of coping motives as a moderator of sadness-drinking relations may be particularly important as self-medication may again be differentiated from alternate affect-drinking mechanisms, in part, by this specific motivation for drinking. Although rarely tested, studies using experience sampling and daily diary methods also suggest that the association between sadness and drinking is stronger in men than in women (Hussong et al., 2001), yet evidence for the moderating role of coping motives in the daily mood-drinking relation is inconsistent (Hussong, et al., 2004; Mohr et al., 2001; Todd, Armeli, Tennen, Carney, \& Affleck, 2003). These studies, however, assessed such vulnerability markers for self-medication assuming a fixed (daily or weekday-weekend) interval between mood and drinking across all individuals. To the extent that shorter or longer intervals between affect and drinking are necessary to detect sadness-drinking relations over individuals, survival analysis may provide a better alternative or adjunctive method to mixed modeling for testing these hypothesized relations.

\footnotetext{
${ }^{2}$ Although contrasting models for affect-drinking relations (i.e., self-medication and a shared etiology model) may act in concert and not be mutually exclusive, testing self-medication within a methodological framework that can best isolate this mechanism from other affect-drinking mechanisms provides an important advantage to understanding the relative influences of each mechanism of risk.
} 
In sum, previous studies of daily assessments show modest mood-drinking covariation as a function of gender and coping motives; however previous analyses also assume a fixed interval across all individuals in the onset of drinking following negative mood. The current study used survival analysis and experience sampling methods to test whether gender and coping motives predicted shorter sadness-to-drinking intervals among those with greater alcohol-related drinking consequences. Moreover, the presence of multiple such vulnerability factors was expected to further elevate risk for a shorter distress-to-drinking interval.

\section{Method}

\subsection{Sample}

A total of 86 participants aged 18-20 were recruited through campus and community advertisement to complete a month long study of coping with stress. ${ }^{3} 84$ of the 86 participants who began the study completed the full protocol. All participants were full-time college students at a large southeastern state university. For the current study, one participant who did not complete a central measure was dropped from analyses, leaving a sample of 85 participants (46\% male, $78 \%$ Caucasian, $16 \%$ African-American, $6 \%$ of other ethnicity, $75 \%$ of participants' parents were college graduates, mean age $=19$ ). With respect to overall drinking patterns, between $78 \%$ and $81 \%$ reported drinking in the past month as compared to $68 \%$ in the national Monitoring the Future college sample (Johnston, O'Malley, \& Bachman, 1999).

\subsection{Procedure}

Participants completed four components of the study. First, participants attended a 1.5-h session at the university involving observational and survey assessments. ${ }^{4}$ Second, participants returned for a similar 1-h session at the end of the 28-day observation period. Third, at the end of each week between this initial and final visit, participants completed a 15-min testing session in which they reported substance use for the past week and received incentives for participation. Fourth, experience sampling of mood and alcohol use occurred on each day between the initial and final visit. Participants were instructed in this protocol at the end of the initial visit when they received a recording booklet and digital pager. Participants received paging signals at random, 2-h intervals between 10 a.m. and 10 p.m. thrice daily for 28 days. When signaled, or as close to the signal as possible, participants completed mood and alcohol use measures in this booklet. If no signal was received by midday, participants contacted the lab and alternate times for making recordings for that day were given. Compliance and equipment failure were assessed each week through phone contact and the weekly assessments at the lab. At the end of the sampling period, $100 \%$ of participants scored their alcohol use ratings as "very honest" and 99\% scored their affect ratings as "very or somewhat honest". All participants were paid for completing each component of the study and a Certificate of Confidentiality was obtained from the U.S. Department of Health and Human Services to protect participant confidentiality.

\subsection{Measures}

1.3.1. Demographic measures-Participant's gender, age and parents' education level were assessed during the initial visit. We used items from Chassin, Rogosch, and Barrera

\footnotetext{
${ }^{3}$ Although not relevant to the current analyses, participants were recruited as friendship pairs, such that the 86 participants were drawn from 43 friendship pairs. Study inclusion criteria thus were having a close friendship of at least 6 months duration and agreement to participate in the 28-day diary study.

${ }^{4}$ Participants completed the initial and final visits as friendship dyads, though all measures were completed independently during these assessments. All other assessments (weekly and daily measures) were completed individually.
} 
(1991) to assess the highest level of mother and father education, with the maximum score of these two items serving as the index of parental education for the current study.

1.3.2. Alcohol-related consequences-In the initial visit, participants completed the short form of the Michigan Alcohol Screening Test (S-MAST; Selzer, Vinokur, \& van Rooijan, 1975; Zung, 1979). The S-MAST presents 13 indicators of problematic alcohol consequences that participants rate as having experienced or not. For the current study, the number of problems endorsed were summed and then trichotomized, due to the skewed distribution of the resulting variable, to reflect having (0) zero, (1) one or (2) two or more alcohol-related consequences. This trichotomized score ranged from zero to two and had a mean of 0.79 , S.D. of .85 , and scale reliability of (Cronbach's a) .76 .

1.3.3. Coping motives-During the initial visit, participants also completed Cooper's Coping and Enhancement motives subscales (Cooper, 1994) to assess reasons for drinking. Five items were used to assess the coping subscale of interest in this study. Participants rated how often they drank for each coping reason using a five-point response scale ranging from (0) almost never or never to (4) almost always or always. Lifetime abstainers were instructed to answer the items based on their beliefs of how often others would drink for these reasons. However, for the current analyses, all motivation scores for participants reporting no alcohol use (i.e., within a 2-month window) were recoded to zero. A sum of the five items was dichotomized, due to the skewed distribution of this predictor and for ease of interpretation, reflecting endorsement of any versus no coping motives (mean=0.69, S.D. $=0.46$ ) ${ }^{5}$ Adequate to strong reliability has been shown for these subscales in previous studies of college students (MacLean \& Lecci, 2000) and was found in the current study (Cronbach's $\mathrm{\alpha}=.83$ ).

A total of $69 \%$ (or $n=59$ out of 85 ) of participants endorsed at least some level of coping motives in the current study. There were no differences between those who did and did not endorse coping motives with regards to levels of sadness at the beginning $[t(83)=-.17, p=$. $86]$ and end $[t(81)=1.47, p=.15]$ of the 28 -day experiencing sampling period nor were there differences in the peak levels of sadness reported during the observation period $[t(83)=.43, p=.67]$. However, as expected, those who endorsed coping motives reported marginally greater alcohol-related consequences [mean $=.54$ for those reporting no coping motives and mean $=.90$ for those reporting coping motives, $t(83)=-1.83, p=.07]$ and significantly greater levels of drinking both at the initial [mean $=1.46 \mathrm{vs}$. mean $=3.58, t(83)=$ $-4.85, p<.001$ ] and final [mean $=1.27$ vs. mean $=3.23, t(81)=-4.84, p<.001]$ assessments of the study period.

1.3.4. Experience sampling-Over the 28 -day period between the initial and final visit, measures of affect were collected three times daily and measures of alcohol use were collected once daily. Measures of alcohol use were modified from Chassin et al. (1991). At the first pager contact of each day, participants reported the number of drinks they had consumed over the past $24 \mathrm{~h}$ using a nine-point response scale ranging from 0 to 8 or more. From these reports, a single daily index of whether or not the participant had used alcohol was formed.

At each of the three daily pager contacts, participants recorded their affect using 59 items from the Positive and Negative Affect Schedule - Expanded Form (PANAS; Clark \& Watson, 1991; Watson \& Clark, 1990). (One item was omitted due to typographical error.) These items were rated as present or absent at the time when the paging signal was received.

\footnotetext{
${ }^{5}$ This variable was also dichotomized using a median split to provide a more even distribution across groups probed in the three-way interaction. However, because results were consistent across both approaches and dichotomizing coping motives as 'none' versus 'any' is more clear conceptually, the latter approach was retained for presentation.
} 
The subscale for sadness was the focus of the current study. To determine the level of sadness over the observation period, items for each scale were summed within each pager report (for each of the three daily pager contacts). The maximum score of peak sadness over all reports (i.e., to index overall level or severity of peak sadness) was then identified as well as the day on which this peak was first observed (i.e., to assess timing of peak sadness). The first peak day of sadness occurring in the observation period was coded as the beginning of the survival period for subsequent analyses (see below). When missing data for daily reports of alcohol use occurred after the first peak day for sadness but before the end of the observation period, we used weekly retrospective reports of the number of days of drinking in a given week to recover these data. When this method did not produce consistent results or particular days could not be recovered with certainty (e.g., when 2 days of missing data occurred within a week and only 1 day of drinking was reported in retrospective weekly reports), data were considered censored at the point of unrecoverable missing data. ${ }^{6}$

\subsection{Analytic approach}

The proposed hypotheses were tested using a series of survival analyses. All analyses were conducted using Cox's Proportional Hazard Model as estimated through PROC PHREG in SAS (Allison, 1995). This method models the hazards of drinking or time until drinking onset (i.e., the likelihood of drinking onset among those who have not yet onset for each day subsequent to baseline). Survival analyses require a restructuring of the dataset. First, the peak level of sadness within each individual's observation period was identified. Second, the first day on which this peak level of sadness occurred was defined as the first day of the interval for analysis. Third, the outcome variable was then defined as the time between the first day of the analytic interval and the first proceeding day on which drinking occurred. For those individuals who did not drink during the observation period following the onset of the analytic interval (42\%), data were considered (right) censored. ${ }^{7}$

Because this study focused on correlates of the interval between peak sadness and drinking as the outcome of interest, several potential confounds that may also serve to shorten this interval must be controlled in the analyses when testing for potential predictors. First, because peak levels of sadness may be evidenced on more than one occasion during the observation period (i.e., more than one maximum score was present), the first peak occurrence of sadness was arbitrarily selected as the first day of the analytic interval. However, the repeated experience of sadness may create a bias in those for whom the length of the interval was misestimated. To address this concern, I included an index of the number of peak sadness days in the observation period as a control variable (mean=3.65, S.D. $=5.96$, range $=1-26$ ). Similarly, I also included an index of the level of the peak sadness because this factor may also systematically index a mis-estimated distress-to-drinking interval (mean $=0.48$, S.D. $=0.28$, range $=0-1$ ). Finally, because participants are more likely to drink for multiple reasons on the weekend (i.e., from Thursday through Saturday nights for college students) than during the week, I included an index of the number of days between the beginning of the survival period (i.e., the first peak day for sadness) and the proceeding weekend (mean $=1.56$, S.D. $=1.55$, range $=0-4)$.

\footnotetext{
${ }^{6}$ Evidence for validity of daily alcohol reports comes from consistency of these reports with weekly and monthly retrospective reports of alcohol use collected from these same participants within the current study.

${ }^{7}$ Those participants with censored data differed little or not at all from those who drank within the observation period following peak sadness on indices of sadness (peak levels of sadness [ $t(83)=1.81, p=.07]$, initial levels of sadness [ $t(83)=1.73, p=.09]$, and final levels of sadness $[t(83)=1.57, p=.12]$; although trend-level differences were in the direction of the censored group having higher sadness). Censored cases reported lower initial alcohol use $[t(83)=-4.63, p<.001]$ and final alcohol use $[t(81)=-7.19, p<.001]$, although they did not differ with respect to alcohol-related consequences $[t(83)=1.20, p=.23]$.
} 


\section{Results}

Two survival analyses were conducted to test the study hypotheses. First, all control variables and the main effects of gender, drinking to cope and alcohol-related consequences were included as predictors of drinking. Control variables included both demographic variables (i.e., participant age, race and parental education) as well as variables related to design features (i.e., the number of peak days of sadness, the overall level of peak sadness and the number of days between the first observed peak day and the next weekend day). Results for this model are presented in Table 1. Both gender and alcohol-related consequences were related to drinking hazards, although coping motives were not. Males and those with lower alcohol-related consequences were more likely to drink sooner following peak sadness. In addition, participants who were older and white were more likely to drink sooner in the days following peak sadness than were younger and ethnic minority participants, respectively.

Given that the effect of alcohol-related consequences is inconsistent with expectations, I further explored these predictors as multiplicative indicators of risk. Specifically, to test whether the predictors of participants' gender, coping motives and alcohol-related consequences together define a group at heightened risk for drinking more immediately after peak levels of sadness, a final survival analysis examined the two- and three-way interactive effects among these three variables in predicting the hazard for drinking. Results are reported in Table 2. A marginally significant three-way interaction among these three predictors was found. To probe this finding, I plotted the survival functions related to the proportional hazard functions. First, eight strata were created to define groups based on participant gender, the presence of coping motives or not, and having zero versus two or more alcohol-related consequences. (Only two levels of consequences are plotted to demonstrate the finding.) I then estimated survival functions using PROC PHREG in SAS for each of these strata (or groups; see Figs. 1 and 2). Resulting plots showed that, for women, greater coping motives and alcohol-related consequences were associated with lower survival (or greater hazard) estimates such that they are more likely than other women to drink sooner after peak days for sadness. For men, lower survival estimates were found for men with coping motives but no alcohol-related consequences, followed by men without either coping motives or alcohol-related consequences, suggesting that these groups of men are more likely to drink sooner after peak days of sadness than are other men. These findings suggest that the pattern of risk factors differ for men and women, with that for women being more consistent with the proposed hypotheses.

Sensitivity analyses were then performed to determine whether findings changed when survival was modeled as time to 'heavy' drinking (consuming five or more drinks in an episode) rather than any drinking and when non-proportional hazards were estimated for each main effect. No changes in key findings were found. I also included interactions between the indicator of timing (i.e., days between the peak sadness and the weekend) and each main effect in the models to determine whether the likelihood of drinking following peak sadness was a function of both main effects and the timing of the peak. No evidence for this hypothesis was found. Finally, I tested whether changing the beginning of the survival interval from peak sadness to the onset of daily data collection produced meaningfully different findings. None of the main effects or interactions involving drinking to cope and alcohol-related consequences was related to drinking in this alternate model, indicating that the prediction of survival is related to the selection of peak sadness as the onset of the distress-to-drinking interval. This final analysis suggests that findings are specific (to the outcome of drinking following peak sadness) rather than general (to the outcome of drinking without reference to predisposing events). Moreover, as an indication that shorter distress-to-drinking intervals were meaningful indicators of drinking 
involvement, quantity of alcohol consumed significantly correlated with the duration from peak levels of sadness to first drinking episode for non-censored cases, with those who drank more quickly after peak sadness also drinking more heavily $(r=-.31, p<.01)$.

\section{Discussion}

The current study is the first to combine survival analysis and daily sampled data to test the self-medication hypothesis in a manner permitting individually varying intervals between days of heightened sadness and subsequent drinking. Specifically, participant gender and coping motives were posited to predict shorter sadness-to-drinking intervals among those with greater alcohol-related drinking consequences. Mixed support for the hypothesis was found. Men drank sooner following peak sadness than did women. However, the main effect of alcohol-related consequences was counter-intuitive, such that individuals reporting lower consequences had shorter distress-to-drinking intervals than did their peers. The three-way interaction among these vulnerability factors indicated that significant gender differences in part accounted for this unexpected finding. Specifically, women, as expected, were more likely to drink in the days immediately following elevated sadness when they also reported being motivated to drink to cope and having experienced alcohol-related consequences. For men, those showing greater risk for drinking in the days following peak sadness did not report alcohol-related consequences, with men who also reported the presence of coping motives showing the greatest risk for drinking after peak sadness.

These results suggest that for both men and women, holding greater coping motives for drinking is manifested as greater risk for drinking in the days following elevated sadness. Coping motives thus not only identify a reason or potential trigger for drinking, but also may suggest a more uncontrolled style of drinking that is less responsive to social controls for drinking. Consistent with this possibility, Cooper, Frone, Russell, and Mudar (1995) suggest that coping, as opposed to enhancement, motives for drinking are more strongly associated with drinking problems. As such, shorter intervals between distress and drinking in those motivated to drink for coping reasons may translate for these college students into more skipped classes or workdays, drinking in more dangerous circumstances, more atypical bouts of weekday drinking, and the accumulation of a heavier drinking peer group that is more accepting of (and perhaps likely to reinforce) these drinking choices. Thus, although coping motives are less commonly endorsed than are social motives in college students (MacLean \& Lecci, 2000), and young drinkers more generally, they may nonetheless identify a group at risk for later problems.

The results of the current study also suggest that the relation between alcohol-related consequences and the risk of drinking after peak sadness differs across gender of the drinker. For men, such consequences predicted lower risk for drinking after sadness whereas the opposite occurred in women. Previous literature suggests that gender differences in the role of depression in adult alcohol use are complex. Studies of adult alcoholics largely show that the comorbidity of alcoholism and secondary depressive disorders defines a subtype of alcoholism for which women show greater risk than do men (Zucker, 1986). However, studies of young adults also report stronger relations between stress/distress and heavy drinking in men than in women (Cooper et al., 1992; Hussong et al., 2001). One explanation for such seemingly contradictory findings is that the strength and implications of the link between distress and drinking depends on whether normal variations or clinical levels of disturbance are the focus of inquiry in defining alcohol use. The current study focuses on sadness and alcohol use rather than on depressive symptoms as a predisposing factor for alcohol disorders, and thus generalization to clinical levels are limited. 
Nonetheless, the current findings suggest that self-medication, or the immediacy of drinking following heightened sadness, may have different consequences across gender. For women, self-medication may have more dire consequences because it enhances the unproductive ruminative style of coping that is more likely to characterize and thus to exacerbate women's depressive styles (Nolen-Hoeksema, Larson, \& Grayson, 1999). Alternatively, a telescoping effect, reported to describe the faster acceleration of women from drinking to problem drinking and abuse as compared to men (Zilberman, Tavares, \& el-Guebaly, 2003), may suggest that the young women in the current study are simply showing alcohol-related consequences as a result of self-medication earlier than their male counterparts.

Longitudinal studies are needed to address this possibility and to further evaluate the implications of this style of drinking for future problems in men.

In conclusion, the current study found that women drank more immediately following sadness when they were both motivated to drink to cope and reported alcohol-related consequences whereas only coping drinking motives identified a more immediate drinking response after elevated sadness in men. Although the current study provides an example of how survival analyses may be used to examine drinking risk within daily assessment methods, it does not address daily covariation in mood and drinking (or consider change in mood prior to drinking episodes) as the question of interest (although see Hussong, Feagans, \& Hersh, 2006; Hussong, Galloway \& Feagans, 2005; Hussong et al., 2001 for examples). Rather, the strength of the current study is in explicitly testing predictors of the time lag between peak sadness and subsequent drinking. Although findings of the current study suggest that survival analysis may be a useful technique for such purposes, the study is limited by a modest sample size and caution is warranted in generalizing findings prior to replication with a larger sample. These limits are offset by the unique approach to analyzing experience sampling data and reliance on repeated assessments of distress and drinking within a short interval offered by the current study.

\section{Acknowledgments}

This research received support from the National Institute on Drug Abuse grants US-NIDA DA15398 and USNIDA DA12912 and a University of North Carolina Research Council Award.

\section{References}

Allison, PD. Survival analysis using the SAS System: A practical guide. Cary, NC: SAS Institute, Inc; 1995.

Armeli S, Tennen H, Affleck G, Kranzler HR. Does affect mediate the association between daily events and alcohol use? Journal of Studies on Alcohol. 2000; 61:862-871. [PubMed: 11188492]

Baker TB, Piper ME, McCarthy DE, Majeskie MR, Fiore MC. Addiction motivation reformulated: An affective processing model of negative reinforcement. Psychological Review. 2004; 111(1):33-51. [PubMed: 14756584]

Chassin L, Rogosch F, Barrera M. Substance use and symptomatology among adolescent children of alcoholics. Journal of Abnormal Psychology. 1991; 100(4):449-463. [PubMed: 1757658]

Clark LA, Watson D. Tripartite model of anxiety and depression: Psychometric evidence and taxonomic implications. Special Issue: Diagnoses, dimensions, and DSM-IV: The science of classification. Journal of Abnormal Psychology. 1991; 100(3):316-336. [PubMed: 1918611]

Cooper ML. Motivations for alcohol use among adolescents: Development and validation of a fourfactor model. Psychological Assessment. 1994; 6(2):117-128.

Cooper ML, Frone MR, Russell M, Mudar P. Drinking to regulate positive and negative emotions - A motivational model of alcohol use. Journal of Personality and Social Psychology. 1995; 69:9901005. [PubMed: 7473043] 
Cooper ML, Russell M, Skinner JB, Frone MR, Mudar P. Stress and alcohol-use - Moderating effects of gender, coping, and alcohol expectancies. Journal of Abnormal Psychology. 1992; 101(1):139_ 152. [PubMed: 1537960]

Flynn HA. Comparisons of cross-sectional and daily reports in studying the relationship between depression and use of alcohol in response to stress in college students. Alcoholism, Clinical and Experimental Research. 2000; 24:48-52.

Greeley, J.; Oei, T. Alcohol and tension reduction. In: Leonard, KE.; Blane, HT., editors. Psychological theories of drinking and alcoholism. New York: Guilford Press; 1999. p. 14-53.

Hussong, AM.; Feagans Gould, L.; Hersh, MA. The relation between negative affect and drinking in adolescence: Delineating vulnerable sub-populations. 2006. Manuscript submitted for publication

Hussong, AM.; Galloway, CA.; Feagans, LA. Coping motives as a moderator of daily mood-drinking covariation. 2004. Manuscript submitted for review

Hussong AM, Galloway CA, Feagans LA. Drinking motives moderate daily mood-drinking covariation. Journal of Studies on Alcohol. 2005; 66:344-353. [PubMed: 16047523]

Hussong AM, Hicks RE, Levy SA, Curran PJ. Specifying the relations between affect and heavy alcohol use among young adults. Journal of Abnormal Psychology. 2001; 110(3):449-461. [PubMed: 11502088]

Johnston, LD.; O'Malley, PM.; Bachman, JG. College students and young adults NIH Publication. Vol. 99-4661. Rockville, MD: National Institute on Drug Abuse; 1999. National survey results on drug use from the Monitoring the Future Study, 1975-1998.

MacLean MG, Lecci L. A comparison of models of drinking motives in a university sample. Psychology of Addictive Behaviors. 2000; 14:83-87. [PubMed: 10822750]

Mohr CD, Armeli S, Tennen H, Carney MA, Affleck G, Hromi A. Daily interpersonal experiences, context, and alcohol consumption: Crying in your beer and toasting good times. Journal of Personality and Social Psychology. 2001; 80:489-500. [PubMed: 11300581]

Nolen-Hoeksema S, Larson J, Grayson C. Explaining the gender difference in depressive symptoms. Journal of Personality and Social Psychology. 1999; 77:1061-1072. [PubMed: 10573880]

Selzer M, Vinokur L, van Rooijan A. A self-administered short Michigan Alcoholism Screening Test (SMAST). Journal of Studies on Alcohol. 1975; 36:117-126. [PubMed: 238068]

Steptoe A, Wardle J. Mood and drinking: A naturalistic diary study of alcohol, coffee and tea. Psychopharmacology. 1999; 141:315-321. [PubMed: 10027513]

Swendsen JD, Tennen H, Carney MA, Affleck G, Willard A, Hromi A. Mood and alcohol consumption: An experience sampling test of the self-medication hypothesis. Journal of Abnormal Psychology. 2000; 109:198-204. [PubMed: 10895557]

Tennen H, Affleck G. The challenge of capturing daily processes at the interface of social and clinical psychology. Journal of Social and Clinical Psychology. 2002; 21(6):610-627.

Todd M, Armeli S, Tennen H, Carney MA, Affleck G. A daily diary validity test of drinking to cope measures. Psychology of Addictive Behaviors. 2003; 17:303-311. [PubMed: 14640826]

Watson, D.; Clark, LA. Unpublished manuscript. Southern Methodist University; 1990. The positive and negative affect schedule - Expanded form.

Zilberman M, Tavares H, el-Guebaly N. Gender similarities and differences: The prevalence and course of alcohol-and other substance-related disorders. Journal of Addictive Diseases. 2003; 22:61-74. [PubMed: 14723478]

Zucker RA. The four alcoholisms: A developmental account of the etiologic process. Nebraska Symposium on Motivation. 1986; 34:27-83. [PubMed: 3498124]

Zung BJ. Psychometric properties of the MAST and two briefer versions. Journal of Studies on Alcohol. 1979; 40(9):845-859. [PubMed: 513776] 


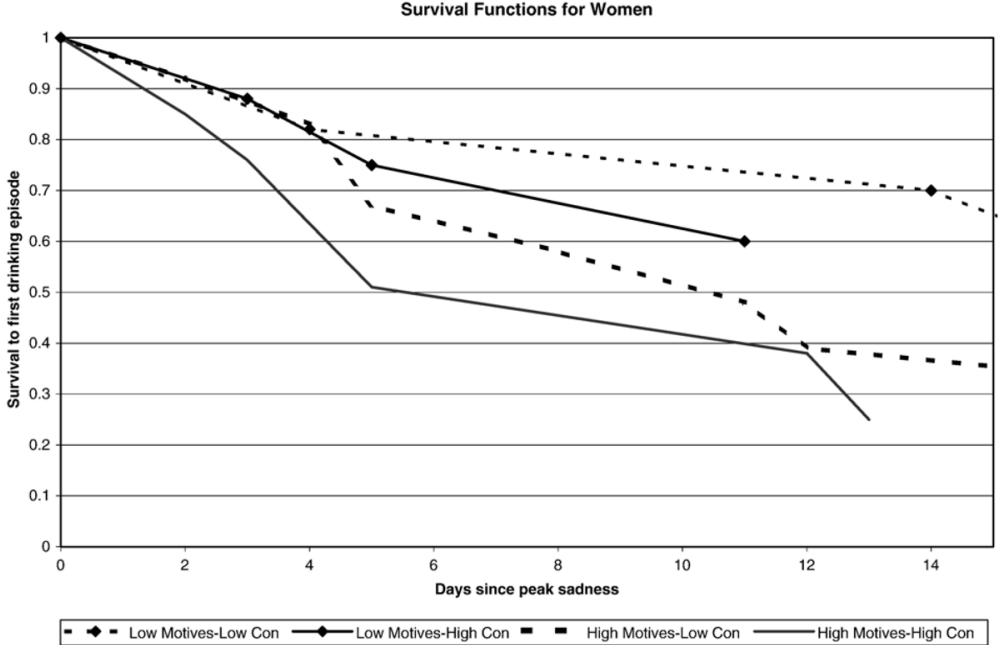

Fig. 1.

Survival functions for women. 


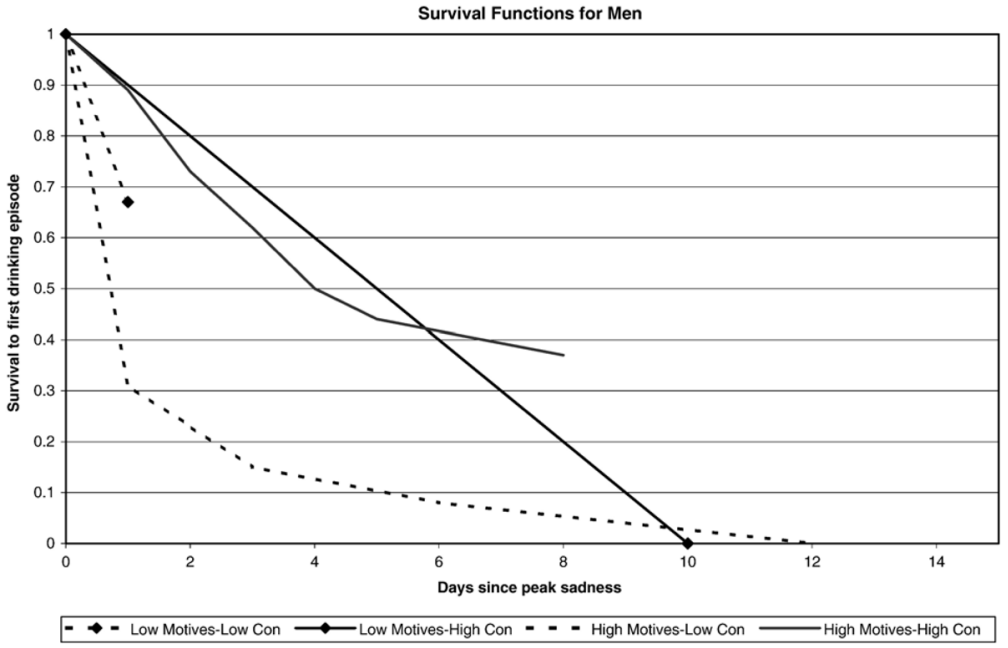

Fig. 2.

Survival functions for men. 
Table 1

Survival analysis of alcohol-related consequences and coping motives

\begin{tabular}{|c|c|c|c|c|}
\hline Predictors & Parameter estimate & Parameter estimate (CI) & $x^{2}$ & Hazard ratio \\
\hline Participant age & 0.60 & $1.10,3.00$ & $5.47^{*}$ & 1.82 \\
\hline Participant race & -1.58 & $0.07,0.58$ & $8.90^{* *}$ & 0.21 \\
\hline Parental education & -0.38 & $0.44,1.07$ & 2.78 & 0.69 \\
\hline Days to weekend & -0.19 & $0.66,1.04$ & 2.64 & 0.83 \\
\hline Peak level of sadness & -0.87 & $0.12,1.51$ & 1.76 & 0.42 \\
\hline Number of peak days & -0.02 & $0.92,1.05$ & 0.25 & 0.98 \\
\hline Participant gender & 1.08 & $1.47,5.89$ & $9.31^{* *}$ & 2.94 \\
\hline Drinking to cope & 0.51 & $0.73,3.82$ & 1.46 & 1.67 \\
\hline Alcohol-related consequences & -0.48 & $0.41,0.94$ & $5.13^{*}$ & 0.62 \\
\hline Model fit & \multicolumn{4}{|c|}{ Wald's $X^{2}(9)=37.58, p<.001$} \\
\hline$p<.05$. & & & & \\
\hline${ }^{* *} p<.01$ & & & & \\
\hline
\end{tabular}


Table 2

Survival analysis of interactive effects of risk predictors

\begin{tabular}{|c|c|c|c|c|}
\hline Predictors & Parameter estimate & Parameter estimate (CI) & $x^{2}$ & Hazard ratio \\
\hline Participant age & 0.55 & $1.05,2.87$ & $4.56^{* * *}$ & 1.73 \\
\hline Participant race & -2.23 & $0.03,0.34$ & $14.64^{* * * *}$ & 0.11 \\
\hline Parental education & -0.45 & $0.39,1.04$ & $3.25^{*}$ & 0.64 \\
\hline Days to weekend & -0.26 & $0.61,0.98$ & $4.46^{* * *}$ & 0.77 \\
\hline Peak level of sadness & -1.01 & $0.10,1.34$ & 2.29 & 0.37 \\
\hline Number of peak days & -0.01 & $0.93,1.06$ & 0.03 & 0.99 \\
\hline Participant gender & 1.43 & $0.43,40.64$ & 1.51 & 4.17 \\
\hline Drinking to cope & 0.59 & $0.54,5.58$ & 0.92 & 1.80 \\
\hline Alcohol-related consequences & 0.45 & $0.51,4.76$ & 0.62 & 1.56 \\
\hline Alcohol-related consequences $\times$ Gender & 0.34 & $0.23,8.51$ & 0.14 & 1.41 \\
\hline Alcohol-related consequences $\times$ Coping motives & -0.18 & $0.24,2.87$ & 0.08 & 0.83 \\
\hline Coping motives $\times$ Gender & 0.79 & $0.20,24.10$ & 0.40 & 2.18 \\
\hline Alcohol-related consequences $\times$ Coping motives $\times$ Gender & -1.89 & $0.02,1.05$ & $3.64^{*}$ & 0.15 \\
\hline Model fit & \multicolumn{4}{|c|}{ Wald's $X^{2}(13)=52.65, p<.001$} \\
\hline \multicolumn{5}{|l|}{${ }^{*}{ }^{<}<.10}$. \\
\hline \multicolumn{5}{|l|}{$* *{ }^{*}<.05}$. \\
\hline$* * * * 0.001$. & & & & \\
\hline
\end{tabular}

